\title{
Local intravascular delivery of low-density-lipoprotein cholesterol corresponds with increased intimal thickening in a healthy porcine coronary model. A prelude to development of a model of atherosclerosis
}

\author{
Piotr P. Buszman ${ }^{1,2}$, Bartlomiej Orlik ${ }^{1}$, Krzysztof P. Milewski ${ }^{1}$, Tomasz Roleder $^{3}$, Michał Jelonek ${ }^{1}$, \\ Filip Polczyk ${ }^{4}$, Frank D. Kolodgie ${ }^{5}$, Maciej Jankowski ${ }^{6}$, Renu Virmani ${ }^{5}$, Paweł E. Buszman ${ }^{1,3}$ \\ ${ }^{1}$ Center for Cardiovascular Research and Development of American Heart of Poland, Katowice, Poland \\ ${ }^{2}$ Andrzej Frycz Modrzewski Krakow University, Krakow, Poland \\ ${ }^{3}$ Medical University of Silesia, Katowice, Poland \\ ${ }^{4}$ Euromedic Ltd, Katowice, Poland \\ ${ }^{5} \mathrm{CV}$ Path Institute, Gaithersburg, MA, USA \\ ${ }^{6}$ Department of Clinical Chemistry, Medical University of Gdansk, Gdansk, Poland
}

Adv Interv Cardiol 2019; 15, 1 (55): 81-90

DOI: https://doi.org/10.5114/aic.2019.83774

\begin{abstract}
A bstract
Introduction: Preclinical, vascular response studies are limited due to lack of underlying disease. The available cholesterol-diet-based and genetic atherosclerotic models are not satisfactory due to long breeding, unpredictable lesion formation, low plaque volume and degree of stenosis.

Aim: To evaluate the vascular response to local, intramural delivery of human, highly atherogenic lipids into healthy domestic swine (DS) coronary arteries.

Material and methods: A total of 24 coronary artery segments of 10 DS were enrolled. Following balloon injury (plain old balloon angioplasty - POBA), segments were assigned to local delivery of $2 \mathrm{ml}$ of human LDL from apheresis ( $400 \mathrm{mg} / \mathrm{dl}, n=9$ ), $0.9 \%$ $\mathrm{NaCl}$ (control, $n=7$ ) or to POBA alone. The solutions were infused with a modified, triple micro-needle catheter into the vessel wall. After 28 days, optical coherence tomography (OCT), virtual histology IVUS (VH-IVUS) and near-infra-red spectroscopy (NIRS) were performed. Following euthanasia, vessel segments were harvested for pathological evaluation.

Results: At 28 days the \% area stenosis in OCT was highest in the LDL group (23.6 \pm 13 vs. $10.8 \pm 7$ vs. $8.1 \pm 7 \% ; p=0.02)$. The presence of necrotic core (LDL: $55.5 \%$, control: $37.5 \%$ and POBA: $42.8 \% ; p=0.77$ ) and dense calcium (LDL: $33.3 \%$, control: $28.5 \%$, POBA: $37.5 \% ; p=0.94)$ in VH-IVUS were comparable between groups. The lipid core burden index in NIRS was negative in all cases. In pathology, the injury was comparable between groups (LDL: $1.6 \pm 0.4$, control: $1.7 \pm 0.8$, POBA: $1.7 ; p=0.8$ ) and specimens showed no signs of necrotic or lipid core. The tissue consisted of smooth muscle cells (SMC)/proteoglycan-rich lesions and inflammatory cells.

Conclusions: Local delivery of saturated human LDL into the coronary artery wall was feasible and resulted in a higher degree of stenosis caused by intimal thickening. A discrepancy between histopathological findings and virtual histology intravascular ultrasound (VH-IVUS) was also noted.
\end{abstract}

Key words: animal model of atherosclerosis, swine, local delivery, in-stent restenosis.

S u m m a ry

We aimed to evaluate the vascular response to local, intramural delivery of human, highly atherogenic lipids into domestic swine (DS). A total of 24 coronary artery segments of 10 DS were enrolled. Following balloon injury (POBA), segments were assigned to local delivery of $2 \mathrm{ml}$ of human $\mathrm{LDL}(n=9), 0.9 \% \mathrm{NaCl}$ (control, $n=7$ ) or to POBA alone. Following 28 days, optical coherence tomography (OCT), virtual histology IVUS (VH-IVUS) and NIRS spectroscopy were performed and vessel segments harvested for evaluation. At 28 days the $\%$ area stenosis in OCT was highest in the LDL group ( $23.6 \pm 13$ vs. $10.8 \pm 7$ vs. $8.1 \pm 7 \% ; p=0.02$ ).

\section{Corresponding author:}

Piotr P. Buszman, Center for Cardiovascular Research and Development of American Heart of Poland, Katowice, Andrzej Frycz Modrzewski Krakow University, Krakow, Poland, e-mail: piotr.buszman@ahop.pl

Received: 5.12.2018, accepted: 27.12.2018. 


\section{Introduction}

Large animal coronary artery models of in-stent restenosis and local drug delivery play an important role in development and validation of novel intravascular technologies and therapies such as vascular stents, scaffolds or drug-coated balloons. They have been validated mainly for drug release kinetics as well as safety by evaluation of healing and biocompatibility [1, 2]. On the other hand, the evaluation of efficacy and translation into the clinical setting has been limited, mainly due to a lack of underlying atherosclerotic lesions. Currently, multiple models of coronary artery disease exist, indicating the possibility of creating thin cap fibroatheromas [3-8]. These models however have multiple disadvantages. Most are based on small laboratory animals, not eligible for testing of human prototype devices. Additionally, the reproducibility, plaque volume, breeding time and cost are not satisfactory. Therefore, with the advances in transcatheter technologies, local delivery methods of lipoproteins supported by high cholesterol diet in large animals have been proposed [9-11] which resulted in positively remodeled, atheromatous but non-occlusive lesions 8-10 weeks following injury. These experiments however were based on high-cholesterol fed diet animals and/or familial hypercholesterolemic swine. Therefore, the independent role of direct cholesterol injection into the vessel wall is unknown.

\section{Aim}

Herein, we sought to evaluate the vascular response after local injection of highly atherogenic human low-density lipoprotein (LDL) in a normocholesterolemic domestic swine coronary model with anticipation of creation of a normocholesterolemic, reproducible model of occlusive atherosclerotic lesions.

\section{Material and methods}

\section{Study design}

After 7-day acclimation, using standard percutaneous techniques, under general anesthesia, coronary angiography with live quantitative analysis (QCA) was performed. One, medial segment of each main coronary artery (LAD, LCX, RCA) without side branches was chosen for the baseline procedure.

Following triple, $130 \%$ balloon overstretch injury (POBA), eligible coronary segments were randomized to three groups: (1) the study group with local delivery of $2 \mathrm{ml}$ of human LDL from apheresis (LDL group); (2) the control group with delivery of $0.9 \% \mathrm{NaCl}$; and (3) the reference group (POBA alone group).

After 28 days of follow-up, control angiography was performed as well as multi-modality intravascular imaging with optical coherence tomography (OCT), virtual histology IVUS (VH-IVUS) and NIRS spectroscopy. Subsequently, the animals were euthanized and the vessel segments were harvested for independent pathological analysis.

\section{Study device}

The solutions were infused into the arterial wall with a modified, triple circumferential micro-needle catheter (Peregrine, Ablative Solutions, San Jose, CA, USA). The standard Peregrine catheter is approved for renal denervation procedures, in which it is used for peri-adventitial alcohol delivery and nerve ablation. The main advantages of these system, such as full proper targeting with full circumferential coverage, efficiency and controlled deployment, have been proved in preclinical studies [12]. In this study, we used a modified Peregrine catheter with a system enabling only short, 1-2 mm micro-needle deployment, sufficient to inject solution into the arterial wall instead of the peri-adventitial space (Figure 1).

\section{Solution - LDL isolation}

Low-density lipoprotein (LDL) was isolated from apheresis waste fluid, obtained from patients with familial hypercholesterolemia undergoing LDL apheresis at the $1^{\text {st }}$ Chair and Clinic of Cardiology, Medical University of Gdansk, by sequential density gradient ultracentrifugation using an ultracentrifuge Beckman Coulter Optima TLX-120 equipped with a fixed angle TLA-100.3 with slow acceleration and deceleration. LDL-apheresis fluid was distributed to $3.5-\mathrm{ml}$ polycarbonate centrifuge tubes and a discontinuous density gradient was made by overlaying the apheresis solution $(2.1 \mathrm{ml})$ with solution containing $\mathrm{NaCl}, 10 \mathrm{mg} / \mathrm{dl}$ EDTA and $1 \mathrm{mM} \mathrm{NaOH}$ (density, $d=1.006$ $\mathrm{g} / \mathrm{ml}$ ). The tubes were ultracentrifuged at $100000 \mathrm{rpm}$ for $1 \mathrm{~h}$ at $4^{\circ} \mathrm{C}$. The upper fraction was discharged and the lower fraction was mixed with $16.7 \% \mathrm{NaCl}$ in the ratio $1: 1$. The high density-adjusted fraction was then centrifuged at $100000 \mathrm{rpm}$ for $2.5 \mathrm{~h}$ at $16^{\circ} \mathrm{C}$ after which the $L D L$ fraction was collected from the top of the tube $(d<1.063 \mathrm{~g} / \mathrm{ml})$. The fraction containing LDL was desalted by running the samples over a Sephadex G-10 column (GE Healthcare) and filtered through a $0.20-\mu \mathrm{m}$ pore-size syringe filter (Corning). Finally, sucrose at a final concentration of $10 \%$ was added and samples were placed at $-80^{\circ} \mathrm{C}$. the LDL concentration was evaluated in a series of samples to confirm the reproducibility of the solutions.

\section{Experimental procedures}

The study protocol has been accepted by the Local Ethics Committee for animal research. All animals received the standard of care outlined in the study protocol and in accordance with the act of animal welfare and the Principles of Care of Laboratory Animals [13]. Swine were fasted overnight before intravascular procedures. All pigs were pre-medicated with atropine $(0.5 \mathrm{mg})$, then sedated with intramuscular ketamine hydrochloride $(20 \mathrm{mg} / \mathrm{kg})$ and xylazine $(2 \mathrm{mg} / \mathrm{kg})$, intubated and anesthetized with 

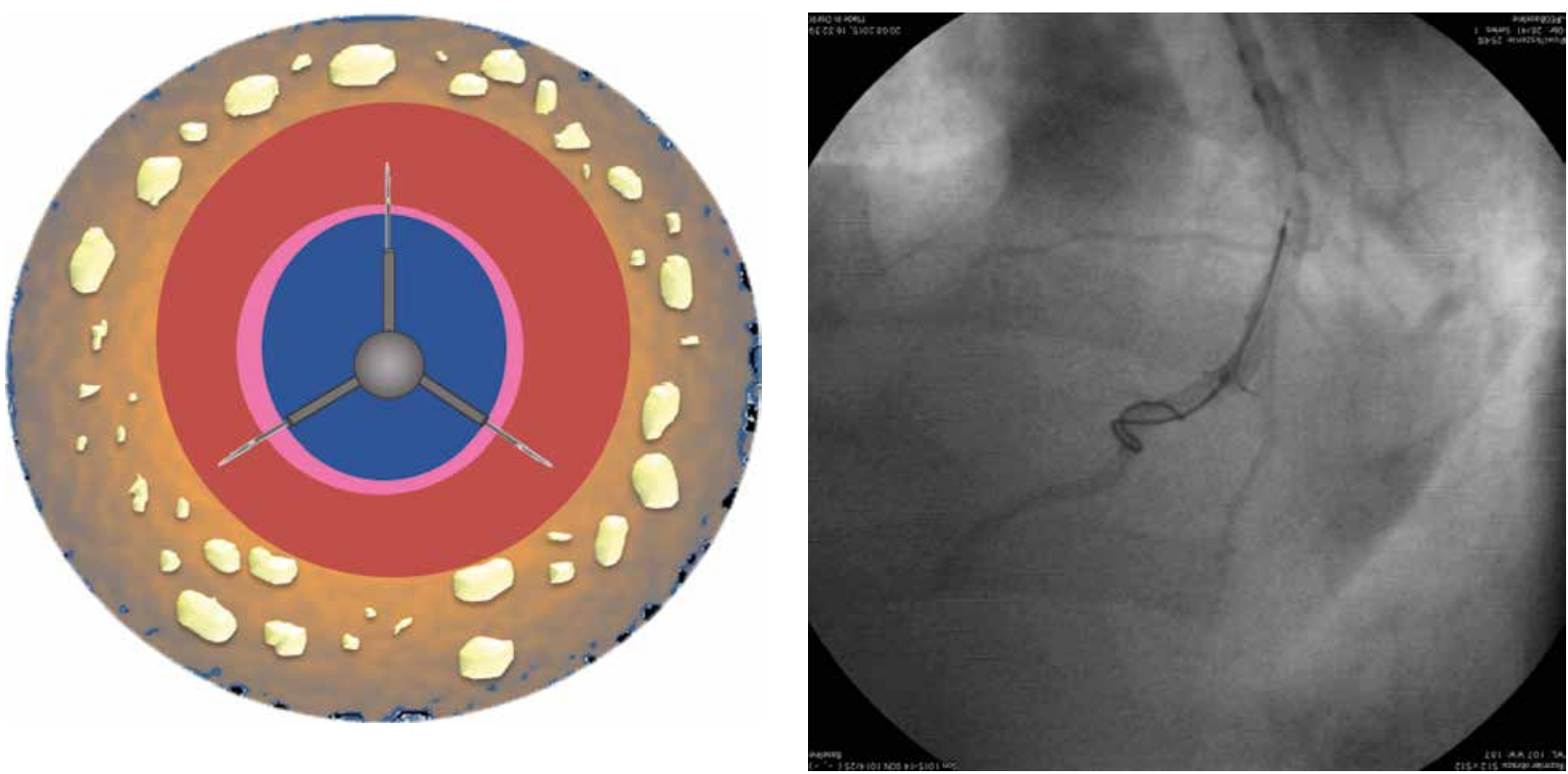

Figure 1. Intramural injection of studied solutions utilizing modified circumferential, triple needle delivery catheter (Peregrine, ASI)

an intravenous propofol bolus (20-40 mg) followed by a continuous infusion $(2-4 \mathrm{mg} / \mathrm{kg} / \mathrm{h})$. The electrocardiogram and blood pressure were continuously monitored during the whole procedure. Arterial access was obtained via the carotid artery utilizing cut-down technique and insertion of a $7 \mathrm{Fr}$ sheath. Anticoagulation with heparin was achieved (3,000-10,000 U) to maintain a coagulation time $\geq 250 \mathrm{~s}$. Following coronary angiography and live QCA analysis, one proximal segment, with diameter of 3-4 mm of each main coronary artery, was chosen for triple balloon dilation (POBA) at an inflation pressure sufficient to achieve $130 \%$ overstretch (balloon-artery ratio). Subsequently, in previously prepared segments, using a modified micro-needle catheter, the LDL solution or $0.9 \% \mathrm{NaCl}$ was injected into the arterial wall. In the reference group, procedures were stopped after POBA. Finally, coronary angiography was performed.

After 28 days of follow-up, under general anesthesia as described above, terminal angiography with QCA, OCT and VH-IVUS was performed. Then all animals were sacrificed with pentobarbital overdose. Their coronary arteries were excised, perfused with formalin solution and then isolated for further histopathological analysis.

\section{Quantitative coronary analysis (QCA)}

Angiography of coronary arteries was performed using a General Electric Innova angiographic unit. A renal, short shaft $7 \mathrm{Fr}$ guiding catheter was used to perform coronary angiography, balloon injury and the needle catheter delivery. QCA analysis was performed in a blinded fashion using QAngio XA Software version 7.1.14.0 (Medis Medical Imaging Systems) from two contralateral projections. The baseline and 28-day follow-up minimal lumen diameters (MLD) and reference vessel diameters (RVD) were taken using the guiding catheter as a standard for measurement for calibration. Subsequently, the balloon-to-artery ratio was calculated. Percent diameter stenosis (\%DS) at follow-up was calculated as: $[1-(M L D / R V D)] \times 100 \%$. Late lumen loss was calculated as MLD at baseline minus MLD at follow-up.

\section{Histological analysis}

Following vessel harvesting, studied segments were immersed in normal buffered formalin 10\% (NBF) and were sent to an independent laboratory for pathological analysis (CVPath, Gaithersburg, MA, USA). All vessel segments were trimmed at three levels each (proximal, middle and distal), processed, embedded in paraffin, sectioned twice serially and the resulting sections were stained with hematoxylin and eosin $(\mathrm{H}+\mathrm{E})$ and Movat's Pentachrome (MP) for evaluation. All sections were analyzed using a scoring system, and the following parameters were calculated: the vessel wall injury, neointima maturity, neointima fibrin, adventitial, media or mural inflammation, endothelialization, the encroachment of the thrombus on the vascular lumen, media hypocellularity, adventitial fibrosis.

There were noted other morphological features such as inflammatory cell type distribution, mural avulsion, endothelium erosion (terminal or artifact), mineralization, media fibrosis, neointima/media/adventitia - necrosis, neointimal vascularization, pre-aneurysmal dilation, occlusion, media smooth muscle cell vacuolation, leukocyte margination, foam cells, media free lipids, hemosiderin/ hemorrhage, laceration/dissection, plaque calcification, fibrous plaque. For these parameters, semi-quantitative scoring at each section level was evaluated as follows: 0 - not present; 1 - present, but minimal feature; 
2 - notable feature, mild; 3 - prominent feature that does not disrupt tissue architecture and is not overwhelming, moderate; 4 - overwhelming feature or feature that effaces or disrupts tissue architecture, severe.

\section{Intravascular imaging \\ OCT image analysis}

The OCT image analysis scrutinized serial cross-sectional images of the vessel, at $1 \mathrm{~mm}$ intervals, using CAAS Intravascular 2.0 software (Pie Medical Imaging BV, Netherlands). The region of interest (ROI) for OCT, IVUS and NIRS analysis was the location of the vessel's injury performed at the index procedure. Plaque composition was analyzed according to previously validated criteria for OCT. In brief, signal-rich homogeneous plaques were classified as fibrous, signal-poor regions with diffuse borders were classified as lipid, signal-poor regions with well-defined borders were classified as calcified plaques, and signal-poor regions with visible media were classified as layered neointima. In addition, the presence of both plaque rupture and/or luminal thrombus was noted during OCT analysis.

Cross-sectional area (CSA), minimal and maximal diameter, and lumen eccentricity ((maximal diameter-minimal diameter)/maximal diameter) of the vessel were measured every $1 \mathrm{~mm}$. The smallest CSA in one segment was taken as the OCT-defined minimal CSA. The OCT reference lumen area was estimated as the largest CSA within $5 \mathrm{~mm}$ proximally or distally - the region of interest (ROI) in the scanned coronary segment.

\section{NIRS image analysis}

The NIRS map analysis was performed using CAAS Intravascular 2.0 software (Pie Medical Imaging BV, Netherlands). The NIRS measurements are demonstrated as a color-coded, red-yellow scaled NIRS map. Each pixel on the NIRS map represents the probability the presence of an atherosclerotic lipid core. Red pixels correspond to low and yellow pixels correspond to a high probability of lipid core. Pixels with insufficient data (e.g. caused by guidewire shadowing) appear as black.

The NIRS map analysis allows calculation of the lipid core burden index (LCBI). The LCBI is estimated by dividing yellow pixels by all pixels (excluding black ones) within the analyzed pullback compartment and are expressed per mill (\%o). In the present study the maximal LCBI was estimated in $4 \mathrm{~mm}$ pullback compartments for every analyzed ROI ( $\left.\max \mathrm{LCBI}_{4 \mathrm{~mm}}\right)$.

\section{IVUS image analysis}

Quantitative grey-scale IVUS measurements were performed every one millimeter in scanned coronary segments using CAAS Intravascular 2.0 software (Pie Medical Imaging BV, Netherlands). Cross-sectional images were quantified for lumen CSA, external elastic lamina (EEM) CSA, plaque and media CSA, plaque burden, plaque area, and plaque volume. The eccentricity of the lumen and EEM CSA was measured as ((maximal diameter-minimal diameter)/maximal diameter). The IVUS reference lumen area was estimated as the largest CSA within $5 \mathrm{~mm}$ located proximally or distally to the ROI in one analyzed coronary segment.

\section{VH-IVUS analysis}

The VH-IVUS analysis was performed at the site of maximum plaque burden within the analyzed ROI. The necrotic core (NC), dense calcium (DC), fibrous fatty (FF) and fibrous tissue $(\mathrm{FI})$ were detected according to previously validated criteria. The VH-IVUS analysis was performed using the Volcano S5 Imaging System.

\section{Statistical analysis}

Normally distributed parametric data are expressed as average and standard deviation, and as median and interquartile range (IQR) in cases of skewed distribution.

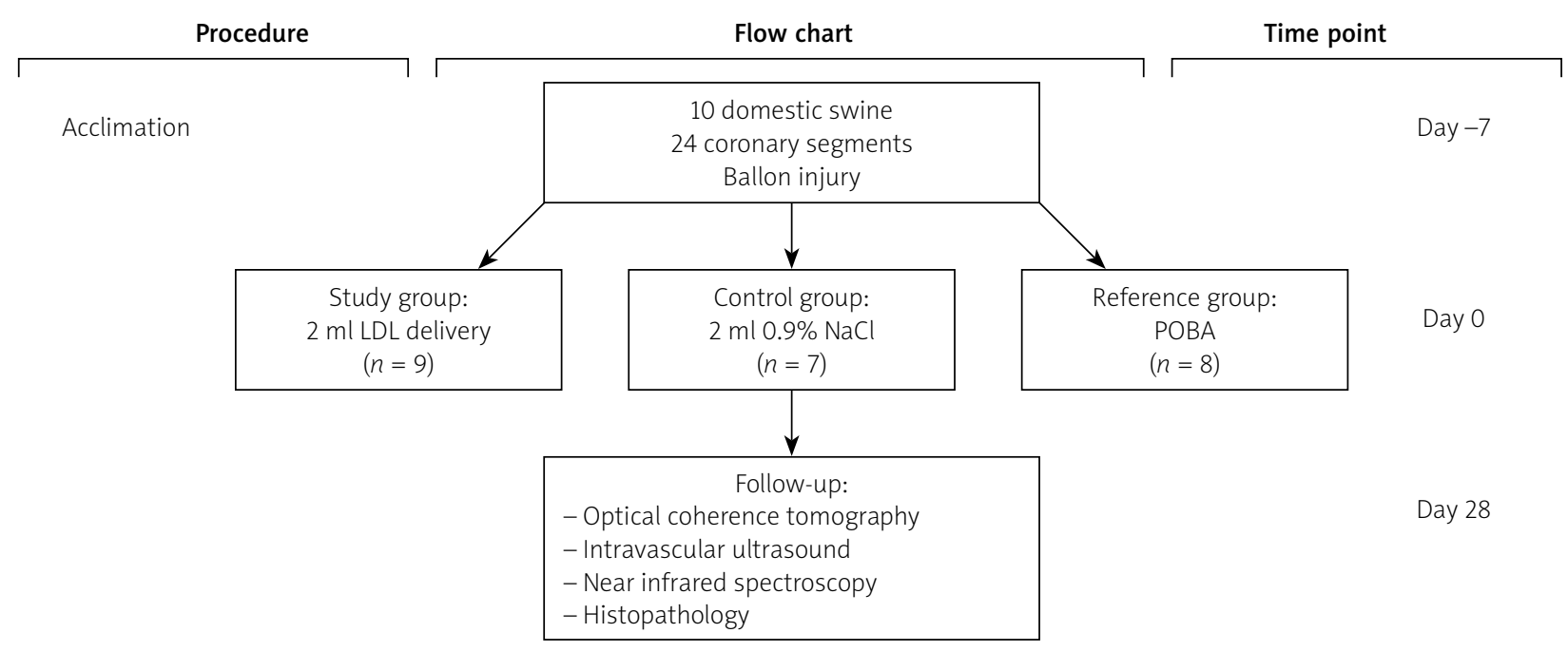

Figure 2. Study flow chart 
When equal variance and normality were observed, oneway analysis of variance (ANOVA) and Student-Newman-Keuls post-ANOVA tests were used to test for differences in variables between stent types. When either the equal variance test or the normality test failed, the Kruskal-Wallis test (with Dunn's method for post-hoc group comparison) was conducted.

\section{Results}

\section{Study flowchart}

A study flowchart is presented in Figure 2. A total of 10 domestic swine, mean age 3-4 months and average weight of $35-45 \mathrm{~kg}$, were included. Six segments were excluded due to vessel size $(n=3)$ and in three cases due to tortuous anatomy delivery of the catheter was not possible. Following randomization 9, 7 and 8 segments were allocated to LDL, $0.9 \% \mathrm{NaCl}$ and POBA groups respectively. There were no periprocedural deaths. All animals survived to terminal follow-up.

\section{Quantitative coronary angiography}

The QCA data are presented in Table I. The baseline vessel reference diameters, balloon diameters and the injury expressed as balloon-to-artery ratios were comparable between groups. At 28-day follow-up no occlusive stenosis ( $<50 \%$ DS) was found in any of the

Table I. Quantitative coronary angiography at baseline and follow-up

\begin{tabular}{|c|c|c|c|c|}
\hline Parameter & $\begin{array}{c}\text { POBA } \\
(n=8) \\
\text { Mean } \pm \text { SD }\end{array}$ & $\begin{array}{c}\text { LDL } \\
(n=9) \\
\text { Mean } \pm \text { SD }\end{array}$ & $\begin{array}{c}0.9 \% \mathrm{NaCl} \\
(n=7) \\
\text { Mean } \pm \mathrm{SD}\end{array}$ & $P$-value \\
\hline \multicolumn{5}{|l|}{ Baseline: } \\
\hline Minimal lumen diameter [mm] & $2.48 \pm 0.3$ & $2.42 \pm 0.2$ & $2.61 \pm 0.4$ & 0.46 \\
\hline Vessel reference diameter [mm] & $2.67 \pm 0.4$ & $2.58 \pm 0.2$ & $2.77 \pm 0.3$ & 0.48 \\
\hline Balloon diameter [mm] & $3.17 \pm 0.2$ & $3.10 \pm 0.2$ & $3.30 \pm 0.2$ & 0.30 \\
\hline Balloon-to-artery ratio & $1.29 \pm 0.2$ & $1.30 \pm 0.2$ & $1.22 \pm 0.2$ & 0.68 \\
\hline \multicolumn{5}{|l|}{ Follow-up: } \\
\hline Minimal lumen diameter [mm] & $2.35 \pm 0.4$ & $2.36 \pm 0.4$ & $2.48 \pm 0.3$ & 0.77 \\
\hline Vessel reference diameter [mm] & $2.53 \pm 0.4$ & $2.61 \pm 0.5$ & $2.75 \pm 0.2$ & 0.55 \\
\hline Percent area stenosis (\%) & $14.45 \pm 6.6$ & $18.11 \pm 6.5$ & $18.57 \pm 11.0$ & 0.58 \\
\hline Percent diameter stenosis (\%) & $7.36 \pm 3.6$ & $9.57 \pm 3.6$ & $9.96 \pm 6.5$ & 0.53 \\
\hline Late lumen loss [mm] & $0.13 \pm 0.4$ & $0.29 \pm 0.9$ & $0.12 \pm 0.4$ & 0.82 \\
\hline
\end{tabular}

Table II. Intravascular ultrasound (IVUS) volumetric, planimetric and near-infra red spectroscopy analysis (NIRS). Optical coherence tomography (OCT) volumetric and planimetric parameters

\begin{tabular}{|c|c|c|c|c|}
\hline Parameter & $\begin{array}{c}\text { POBA } \\
(n=8) \\
\text { Mean } \pm \text { SD }\end{array}$ & $\begin{array}{c}\text { LDL } \\
(n=9) \\
\text { Mean } \pm \text { SD }\end{array}$ & $\begin{array}{c}0.9 \% \mathrm{NaCl} \\
(n=7) \\
\text { Mean } \pm \text { SD }\end{array}$ & $P$-value \\
\hline \multicolumn{5}{|l|}{ Optical coherence tomography: } \\
\hline Minimal lumen area $\left[\mathrm{mm}^{2}\right]$ & $5.54 \pm 1.1$ & $4.31 \pm 2.4$ & $6.66 \pm 2.7$ & 0.15 \\
\hline Minimal lumen diameter (average) & $2.61 \pm 0.3$ & $2.26 \pm 0.7$ & $2.86 \pm 0.7$ & 0.17 \\
\hline Lumen volume & $151.3 \pm 43$ & $143 \pm 72$ & $159 \pm 60$ & 0.89 \\
\hline Minimal lumen diameter & $2.34 \pm 0.2$ & $1.98 \pm 0.6$ & $2.60 \pm 0.7$ & 0.13 \\
\hline \% Diameter stenosis & $12.35 \pm 11.9$ & $35.97 \pm 20.8$ & $16.42 \pm 13.2$ & 0.02 \\
\hline \%AS (MLD based) & $8.13 \pm 7.8$ & $23.67 \pm 13.7$ & $10.80 \pm 8.7$ & 0.02 \\
\hline Lesion length & $20.95 \pm 4.2$ & $21.84 \pm 4.3$ & $18.60 \pm 1.8$ & 0.34 \\
\hline \multicolumn{5}{|l|}{ Intravascular ultrasound and NIRS: } \\
\hline Lumen volume $\left[\mathrm{mm}^{3}\right]$ & $180.6 \pm 101$ & $165.6 \pm 71$ & $198.2 \pm 60$ & 0.72 \\
\hline Lipid core burden index (LCBI, NIRS) & $2.8 \pm 7$ & 0 & 0 & 0.35 \\
\hline Minimal lumen plaque burden & $32.3 \pm 12$ & $38.8 \pm 19$ & $28.6 \pm 17$ & 0.45 \\
\hline Plaque area & $3.1 \pm 2$ & $4.2 \pm 3$ & $2.9 \pm 1$ & 0.43 \\
\hline Plaque volume NIRS' & $55.6 \pm 45$ & $68.5 \pm 41$ & $45.0 \pm 11$ & 0.46 \\
\hline Lumen eccentricity NIRS' & 0.1 & 0.1 & 0.1 & 0.19 \\
\hline
\end{tabular}


vessels and the minimal lumen diameters were similar among groups. Late lumen loss was comparable between groups.

\section{Optical coherence tomography}

The results of the intravascular imaging are presented in Table II and highlighted in Figure 3. The minimal lumen diameters and areas were lowest in the LDL group, although not significantly statistically. The vessel stenosis was highest in the LDL group as represented by the

\section{A}

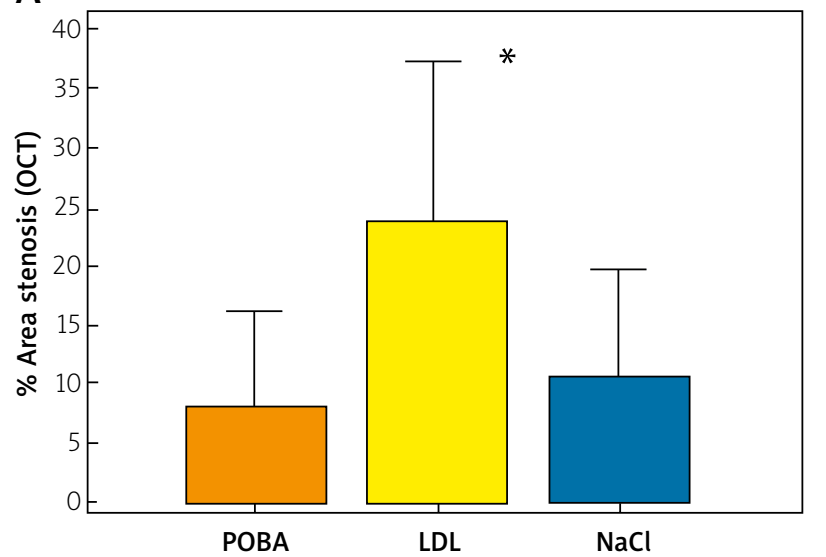

C

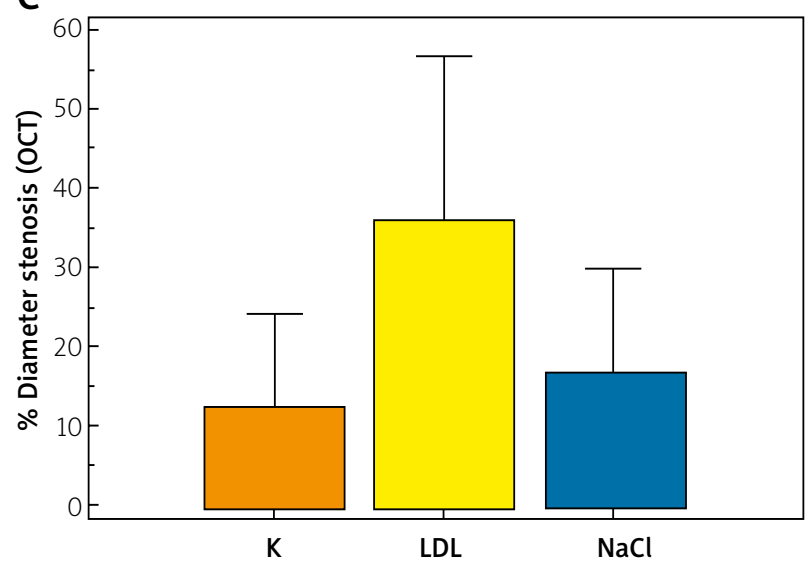

$E$

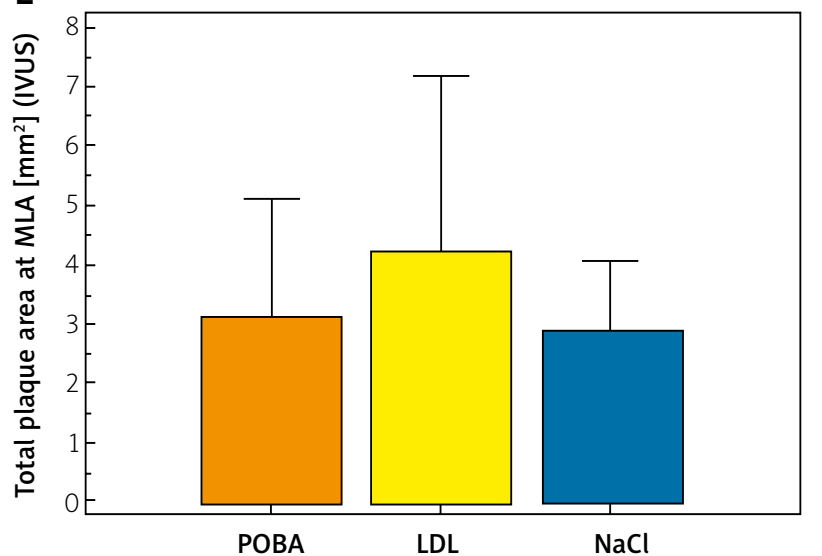

percent area (\%AS) and diameter stenosis (\%DS); it was 2- and 3-fold higher compared to the saline and POBA groups respectively $(p=0.02)$.

\section{Intravascular ultrasound with NIRS and VH}

The plaque burden expressed as plaque volume and area was highest in the LDL group, but there was no statistically significant difference between groups. Similarly, lumen volume was lowest in the study group but without significant differences between groups.

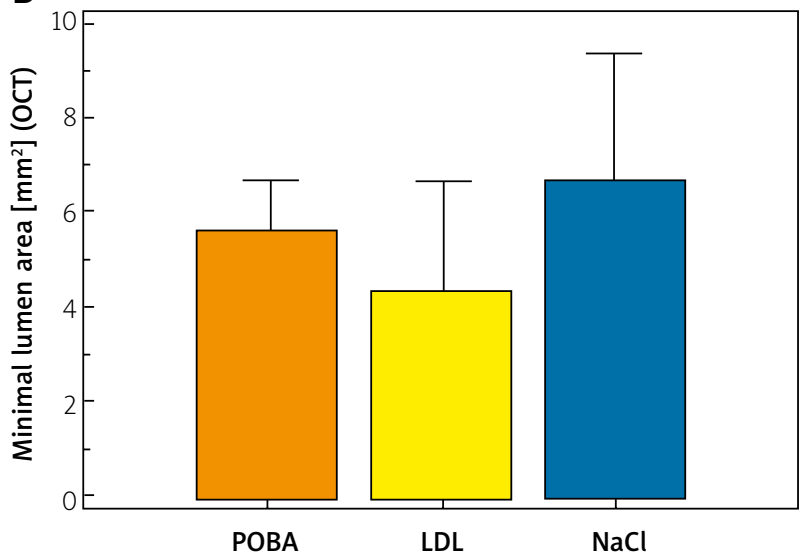

D

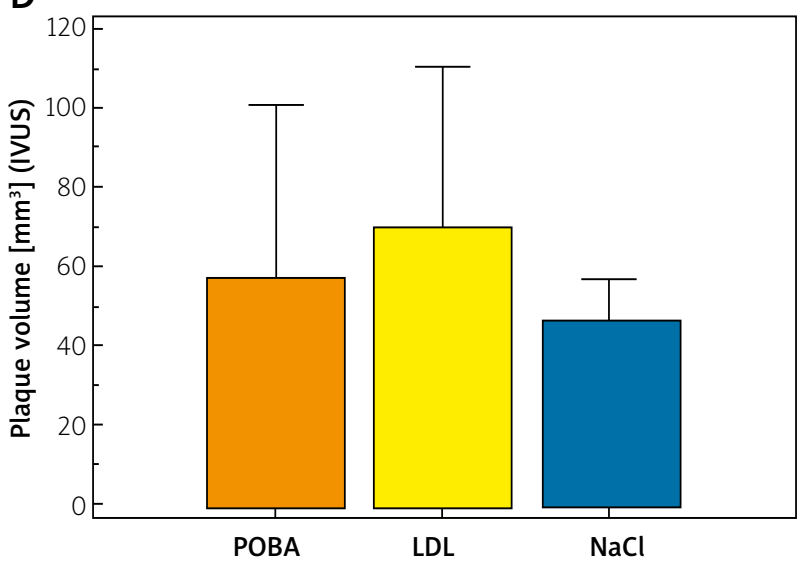

$\mathbf{F}$

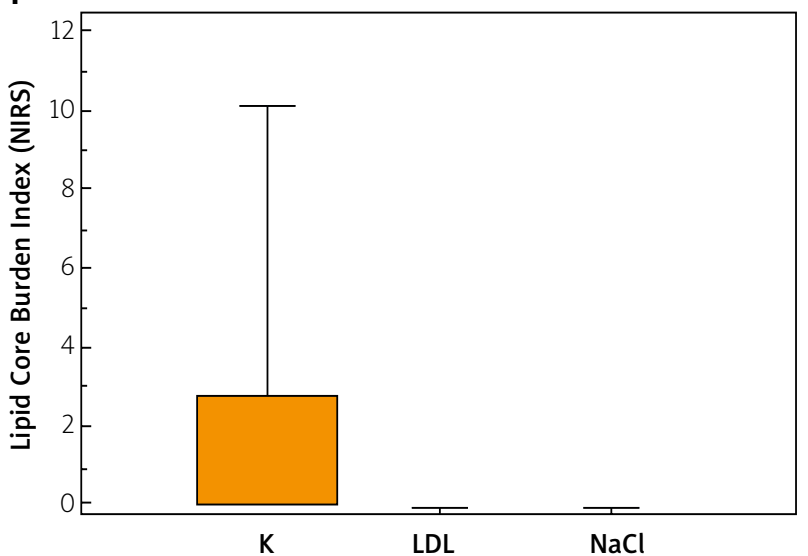

Figure 3. Morphometric lesion evaluation in optical coherence tomography ${ }^{*} P<0.05 L D L$ vs. POBA and $L D L$ vs. $\mathrm{NaCl}$. 
The analysis with near infrared spectroscopy did not show any meaningful signs of lipids in any of the groups as represented by the minimal or zero value of the lipid core burden index.

The virtual histopathology on the other hand showed a differentiated lesion composition including fibrous, fibro-fatty and necrotic core tissue, without any differences between groups (Figure 4).

Representative corresponding cross sections in intravascular imaging and pathology are presented in Figure 5.

\section{Pathological evaluation}

No histologic differences were noted across test and control groups except a tendency toward higher percent area stenosis in the LDL group (Figure 5). The main finding was varying degrees of balloon injury with the typical SMC/proteoglycan-rich lesions observed in most sections, which were considered a fibrotic 'sclerotic' response to varying degrees of underlying balloon injury. There was only rare evidence of macrophage foam cells and general absence of necrotic core formation or oth-
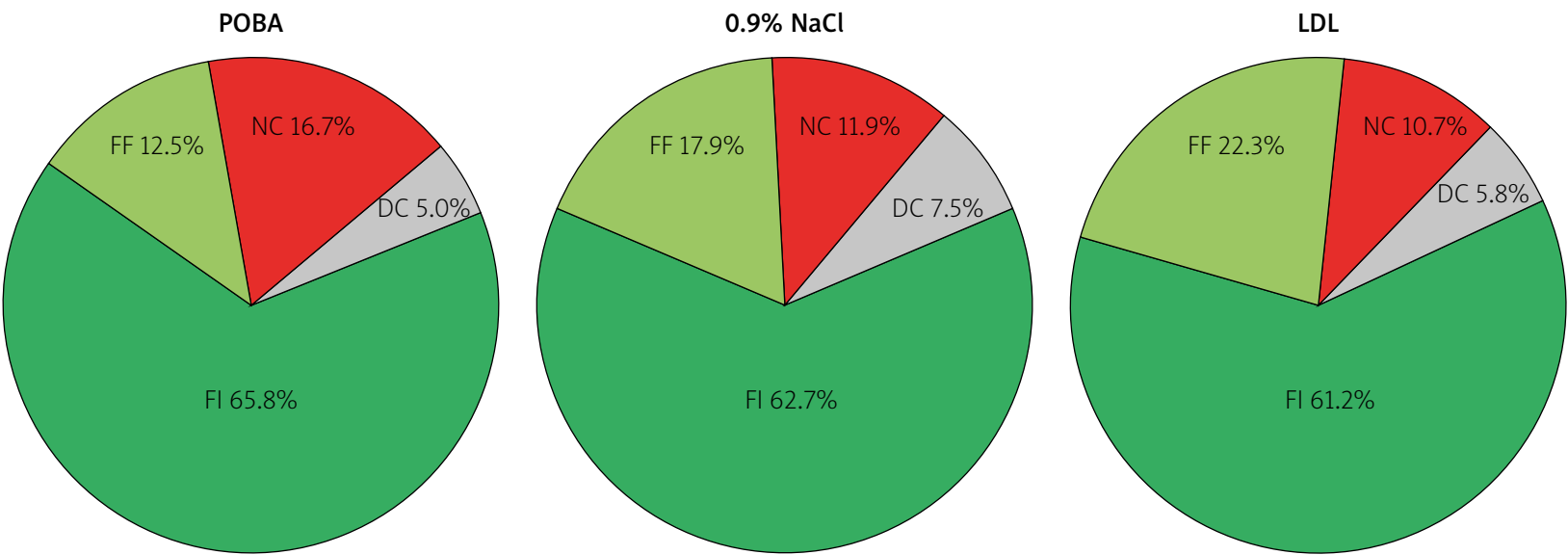

Figure 4. Plaque composition in virtual histology IVUS FI-fibrous tissue, FF-fibrofatty tissue, NC-necrotic core.

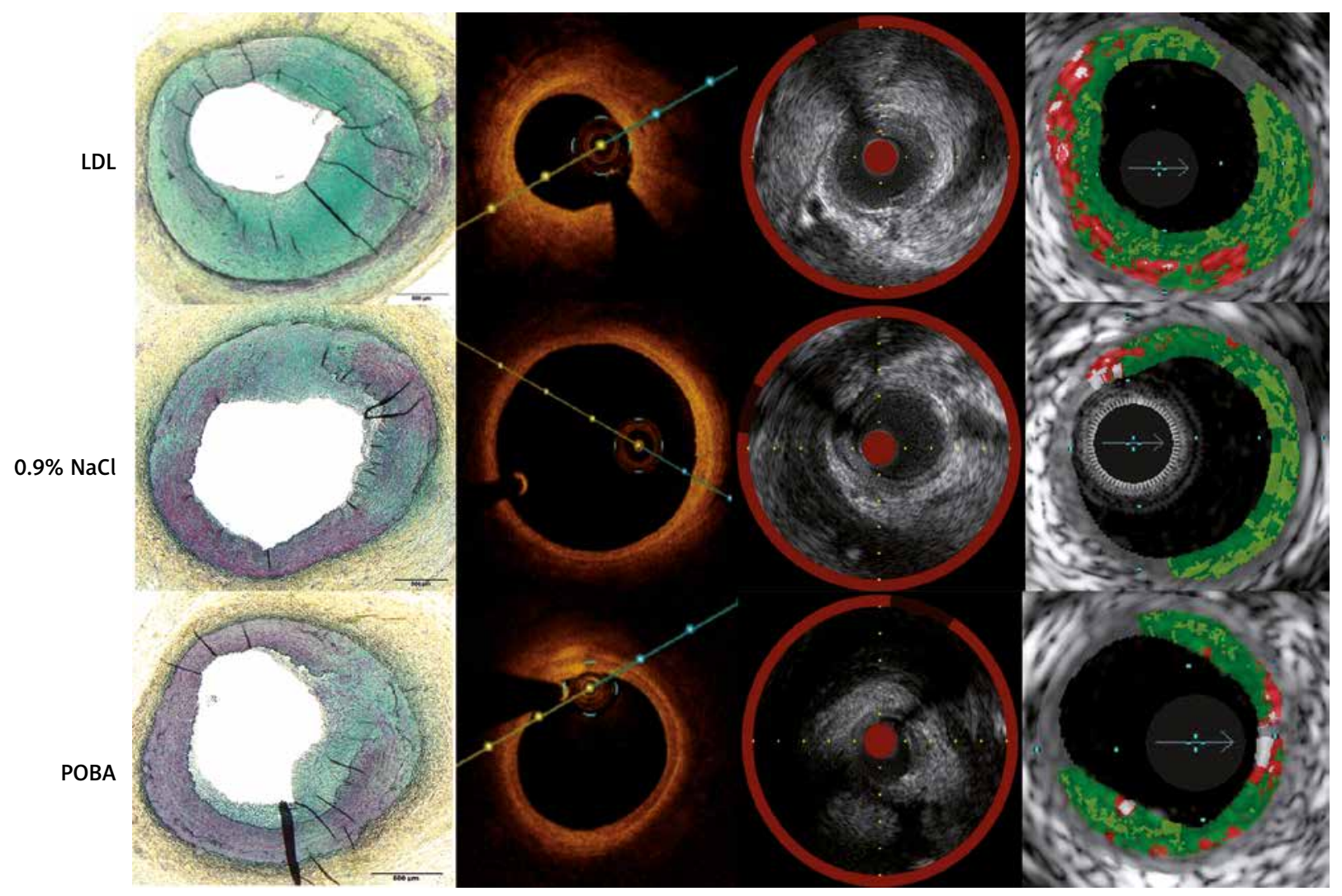

Figure 5. Representative, corresponding images of vessel cross sections in pathology and multimodal intravascular imaging modalities. From left to right: pathology, OCT, NIRS with IVUS, VH-IVUS 
er relevant attributes of atherosclerosis. Inflammation, when present, consisted of lymphocytes, macrophages, and giant cells, and was generally confined to areas of deep injury involving the outer media, adventitia, and peri-adventitial space (Figure 6). Occasional vessels with moderate to severe balloon injury showed lymphoid aggregates with granulomatous-type reactions with notable giant cells mainly in the adventitial or peri-adventitial. Less frequently, eosinophils and plasma cells were also present. A summary of qualitative pathological analysis is presented in Table III.

\section{Discussion}

In the current study, we aimed to develop a reproducible and predictable model of atherosclerosis in a healthy swine model by means of injection of highly saturated human LDL cholesterol into the coronary artery vessel wall. Following 28 days of observation, multiple intravascular imaging modalities were performed to assess the vascular response. Following in-vivo imaging, vessels were harvested for independent pathological analysis. The results showed higher degrees of stenosis in intravascular ultrasound and optical coherence tomography in the group where LDL cholesterol was injected. In the pathological evaluation the SMC/proteoglycan-rich lesions were observed in most sections and were considered as a fibrotic 'sclerotic' response to varying degrees of underlying balloon injury, with some evidence of macrophage foam cells and general absence of necrotic core formation or other relevant attributes of mature atherosclerosis. A tendency toward increased area of stenosis caused by neointimal thickening was observed, thus confirming the intravascular imaging findings. Similarly, previous studies have questioned the validation of the VH-IVUS with pathological evaluation [10].

Multiple reports have been published previously in which the authors anticipated induction of a local atherosclerotic process either with utilization of familial hypercholesterolemic swine or increased cholesterol diet [10, 14]. Both of these models produce advanced atheromas, with lipid pools and necrotic cores, but they mostly induce positive vascular remodeling rather than negative, and the location of these lesions is unpredictable and uncontrollable. Therefore, with the advances in endovascular catheter technologies, local, intramural injection of concentrated lipid solutions has been proposed by Granada et al. $[9,11,15]$. These results have shown various degrees of atherosclerosis progression, but still no significant ste-
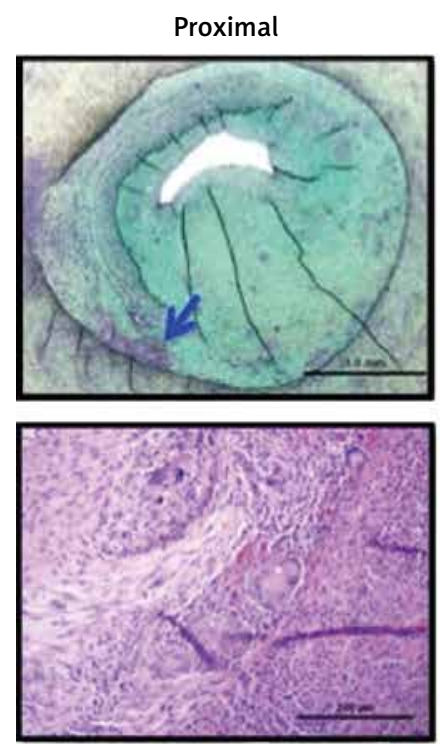

Mid.-distal
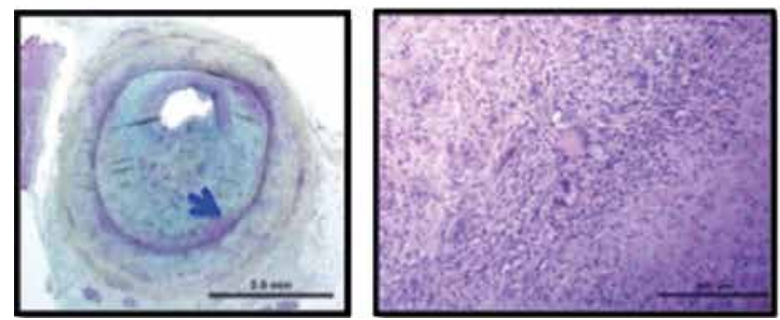

Proximal - mid
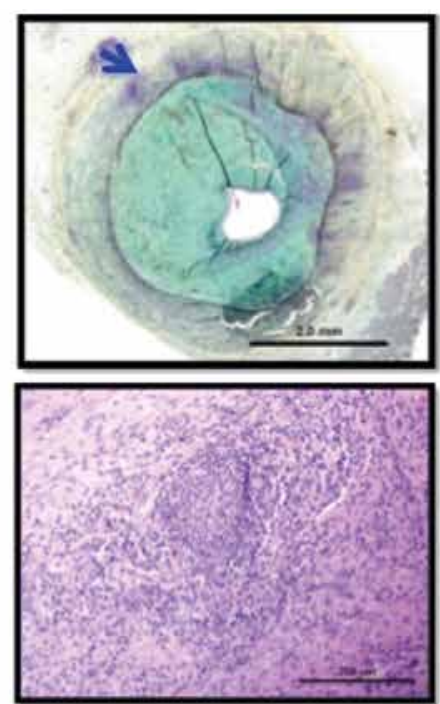

Distal

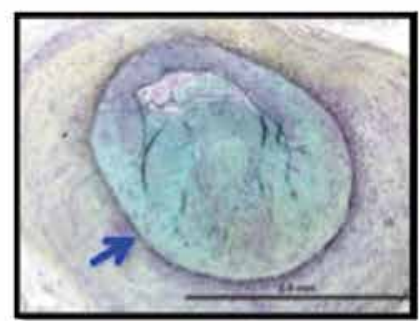

Mid.
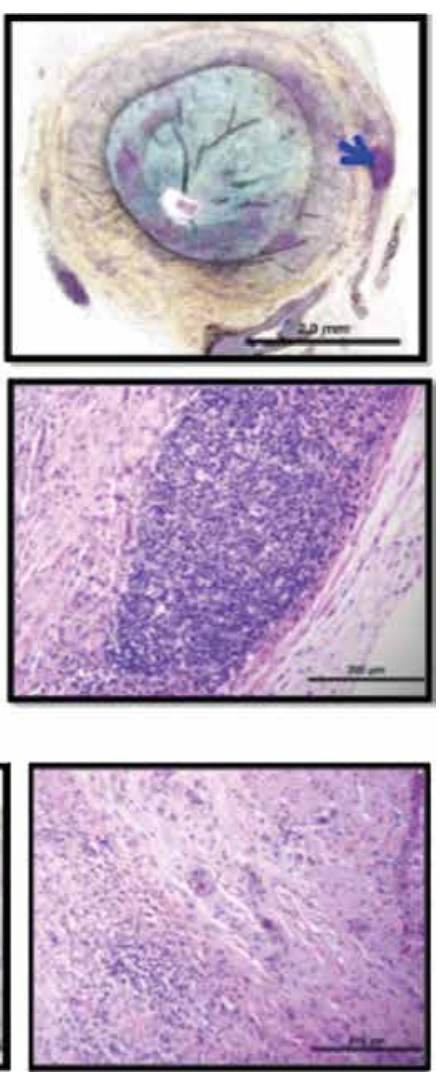

Figure 6. LDL injection - low (Movat pentachrome) and respective high power (H\&E) stained sections show severe arterial balloon injury and restenosis consisting mainly of SMCs and proteoglycan matrix. There are isolated areas of lymphohistiocytic inflammation, including a few giant cells in the deep media, adventitia, and peri-adventitial space (blue arrows) 
Table III. Pathological evaluation

\begin{tabular}{|c|c|c|c|c|c|c|c|}
\hline \multirow[t]{2}{*}{ Parameter } & \multicolumn{2}{|c|}{ POBA $(n=8)$} & \multicolumn{2}{|c|}{$\operatorname{LDL}(n=9)$} & \multicolumn{2}{|c|}{$0.9 \% \mathrm{NaCl}(n=7)$} & \multirow[t]{2}{*}{$P$-value } \\
\hline & Median & $25-75$ & Median & $25-75$ & Median & $25-75$ & \\
\hline \multicolumn{8}{|l|}{ Morphometry: } \\
\hline Lumen area & 1.00 & $0.7-1.3$ & 0.81 & $0.6-1.1$ & 0.91 & $0.5-1.5$ & 0.57 \\
\hline IEL area & 1.93 & $1.3-2.1$ & 1.56 & $1.2-2.2$ & 1.51 & $1.0-2.2$ & 0.97 \\
\hline Med area & 1.93 & $1.6-2.4$ & 2.04 & $1.6-2.8$ & 1.99 & $1.7-2.7$ & 0.97 \\
\hline EEL area & 3.87 & $2.8-4.6$ & 3.72 & $3.0-5.0$ & 3.92 & $2.8-5.1$ & 0.97 \\
\hline Neointimal area & 0.6 & $0.4-1.1$ & 0.97 & $0.4-1.3$ & 0.55 & $0.3-0.6$ & 0.72 \\
\hline Med Inj Score & 1.60 & $1.1-2.1$ & 2.20 & $1.4-3.1$ & 1.80 & $0.9-2.0$ & 0.22 \\
\hline \% Stenosis & 17.20 & $11.7-24.0$ & 33.0 & $14-45$ & 19.0 & $4-24$ & 0.14 \\
\hline \multicolumn{8}{|l|}{ Qualitative analysis: } \\
\hline Free cholesterol & 0.00 & $0.0-0.0$ & 0.00 & $0.0-0.0$ & 0.00 & $0.0-0.0$ & \\
\hline Fibrin/thrombus & 0.00 & $0.0-0.0$ & 0.00 & $0.0-0.0$ & 0.00 & $0.0-0.0$ & \\
\hline Necrotic core & 0.00 & $0.0-0.0$ & 0.00 & $0.0-0.0$ & 0.00 & $0.0-0.0$ & \\
\hline Neo/fibrosis & 0.90 & $0.7-1.0$ & 1.00 & $0.8-1.0$ & 0.80 & $0.6-1.0$ & 0.30 \\
\hline Macrophage & 0.00 & $0.0-0.0$ & 0.02 & $0.0-0.4$ & 0.00 & $0.0-0.0$ & 0.4 \\
\hline T cells & 0.00 & $0.0-0.0$ & 0.00 & $0.0-0.0$ & 0.00 & $0.0-0.0$ & \\
\hline $\mathrm{IPH}$ & 0.00 & $0.0-0.0$ & 0.00 & $0.0-0.0$ & 0.00 & $0.0-0.0$ & \\
\hline Calcification & 0.00 & $0.0-0.0$ & 0.00 & $0.0-0.0$ & 0.00 & $0.0-0.0$ & \\
\hline
\end{tabular}

IEL - internal elastic lamina, Med. - medial layer area, EEL - external elastic lamina.

notic or occlusive lesions were induced. Furthermore, it was impossible to discriminate the independent effect of local injection of cholesterol from generalized hypercholesterolemia. Therefore, in the current study we aimed to evaluate the independent vascular response to cholesterol injection. Moreover, for the first time highly saturated human LDL from apheresis was chosen as a solution and a novel, triple microneedle catheter instead of one needle catheter. To reduce the bias of local vascular injury with balloon and needle injection a placebo controlled group was included. The results showed that in the saturated LDL group injection, vessel stenosis was highest (36\% DS), as shown in OCT evaluation. Therefore, although not significant or occlusive, these lesions can be considered as moderate severity. In pathology, the groups did not differ with regard to tissue composition and consisted mainly of excessive neointimal formation with a trend toward highest neointimal area in the study group. The morphometric calculation in post-mortem pathology sections however is not reliable, due to lack of blood pressure and tissue contractility; therefore in-vivo intravascular imaging provides better vessel morphometric evaluation.

The mechanism of increased neointimal thickening after LDL injection is unclear. We hypothesize that the higher stenosis and neointimal hyperplasia, with SMC and proteoglycan rich lesions, which were observed after LDL injection could be a similar process to intimal thickening in humans. Such a lesion (AHA type III) represents the early stage of atherosclerotic lesion development in humans found during autopsies [16, 17]. Although we did not see lipid pools, typical for pathological intimal thickening (PIT), we believe that this reaction is a footprint after early lipid injection into the vessel wall which initiated the inflammation and excessive neointimal hyperplasia as a consequence. The lack of lipid pools is most likely caused by only single delivery of cholesterol and fast porcine metabolism. Most likely earlier follow-up would have shown lipid deposits. Also, an alternative approach of repeated LDL administration or long-term retention could lead to an immune response which could in turn contribute to more rapid plaque formation. This hypothesis seems to be proven by the latest paper by Nakagawa and Nakashima, who reported that pathologic intimal thickening in human atherosclerosis is formed by accumulation of plasma-derived lipids and dispersion of intimal smooth muscle cells [17]. To fully prove this, further studies and evidence are required.

Validation of the current model with intravascular implantable and drug-eluting devices is required. Furthermore, achieving long-term intramural lipid retention 
should be attempted to provide continuous inflammation, and further and non-reversible development of atherosclerotic lesions.

\section{Acknowledgments}

This paper has been funded by the National Science Center Grant No. 2013/11/D/N25/0382 Poland. Supplementary funding has been provided from the Adamed Scientific Grant.

\section{Conflict of interest}

The authors declare no conflict of interest.

\section{References}

1. Schwartz RS, Edelman E, Virmani R, et al. Drug-eluting stents in preclinical studies: updated consensus recommendations for preclinical evaluation. Circ Cardiovasc Interv 2008; 1: 143-53.

2. Schwartz RS, Edelman ER, Carter A, et al. Preclinical evaluation of drug-eluting stents for peripheral applications: recommendations from an expert consensus group. Circulation 2004; 110: 2498-505.

3. Buja LM, Kita T, Goldstein JL, et al. Cellular pathology of progressive atherosclerosis in the WHHL rabbit. An animal model of familial hypercholesterolemia. Arteriosclerosis 1983; 3: 87-101.

4. Reddick RL, Zhang SH, Maeda N. Atherosclerosis in mice lacking apo E. Evaluation of lesional development and progression. Arterioscler Thromb 1994; 14: 141-7.

5. Schwartz SM, Galis ZS, Rosenfeld ME, Falk E. Plaque rupture in humans and mice. Arterioscler Thromb Vasc Biol 2007; 27: 705-13.

6. Shiomi M, Fan J. Unstable coronary plaques and cardiac events in myocardial infarction-prone Watanabe heritable hyperlipidemic rabbits: questions and quandaries. Curr Opin Lipidol 2008; 19: 631-6.

7. Shiomi M, Ito T, Tsukada T, et al. Cell compositions of coronary and aortic atherosclerotic lesions in WHHL rabbits differ. An immunohistochemical study. Arterioscler Thromb 1994; 14: 931-7.

8. Zadelaar S, Kleemann R, Verschuren L, et al. Mouse models for atherosclerosis and pharmaceutical modifiers. Arterioscler Thromb Vasc Biol 2007; 27: 1706-21.

9. Granada JF, Moreno PR, Burke AP, et al. Endovascular needle injection of cholesteryl linoleate into the arterial wall produces complex vascular lesions identifiable by intravascular ultrasound: early development in a porcine model of vulnerable plaque. Coron Artery Dis 2005; 16: 217-24.

10. Granada JF, Wallace-Bradley D, Win HK, et al. In vivo plaque characterization using intravascular ultrasound-virtual histology in a porcine model of complex coronary lesions. Arterioscler Thromb Vasc Biol 2007; 27: 387-93.

11. Tellez A, Schuster DS, Alviar C, et al. Intramural coronary lipid injection induces atheromatous lesions expressing proinflammatory chemokines: implications for the development of a porcine model of atherosclerosis. Cardiovasc Revasc Med 2011; 12: 304-11.

12. Fischell TA, Vega F, Raju N, et al. Ethanol-mediated perivascular renal sympathetic denervation: preclinical validation of safety and efficacy in a porcine model. EuroIntervention 2013; 9: 140-7.

13. Institute of Laboratory Animal Resources NRC. Principles of Care of Laboratory Animals. NIH Publication 1996; 85-23.
14. Hasler-Rapacz J, Ellegren H, Fridolfsson AK, et al. Identification of a mutation in the low density lipoprotein receptor gene associated with recessive familial hypercholesterolemia in swine. Am J Med Genet 1998; 76: 379-86.

15. van Vlijmen BJ, van den Maagdenberg AM, Gijbels MJ, et al. Diet-induced hyperlipoproteinemia and atherosclerosis in apolipoprotein E3-Leiden transgenic mice. J Clin Invest 1994; 93: 1403-10.

16. Kolodgie FD, Burke AP, Nakazawa G, Virmani R. Is pathologic intimal thickening the key to understanding early plaque progression in human atherosclerotic disease? Arterioscler Thromb Vasc Biol 2007; 27: 986-9.

17. Nakagawa K, Nakashima Y. Pathologic intimal thickening in human atherosclerosis is formed by extracellular accumulation of plasma-derived lipids and dispersion of intimal smooth muscle cells. Atherosclerosis 2018; 274: 235-42. 